\title{
Cyclic brightening in the short-period WZ Sge-type cataclysmic variable SDSS J080434.20+510349.2 ${ }^{\star}, \star \star$
}

\author{
S. V. Zharikov ${ }^{1}$, G. H. Tovmassian ${ }^{1}$, V. V. Neustroev ${ }^{2}$, R. Michel ${ }^{1}$, C. Zurita ${ }^{1}$, J. Echevarría ${ }^{3}$, \\ I. F. Bikmaev ${ }^{4}$, E. P. Pavlenko ${ }^{5}$, Y.-B. Jeon ${ }^{6}$, G. G. Valyavin ${ }^{6}$, and A. Aviles ${ }^{1}$ \\ ${ }^{1}$ Observatorio Astronómico Nacional SPM, Instituto de Astronomía, Universidad Nacional Autónoma de México, Ensenada, BC, \\ México \\ e-mail: zhar@astrosen.unam.mx \\ 2 Centre for Astronomy, National University of Ireland, Galway, Newcastle Rd., Galway, Ireland \\ 3 Instituto de Astronomía, Universidad Nacional Autónoma de México, D.F., México \\ 4 Department of Astronomy, Kazan State University, Russia \\ 5 Crimean Astrophysical Observatory, Nauchny, Ukraine \\ ${ }^{6}$ Bohyunsan Optical Astronomy Observatory, South Korea
}

Received 4 March 2008 / Accepted 23 March 2008

ABSTRACT

\begin{abstract}
Aims. We observed a new cataclysmic variable (CV) SDSS J080434.20+510349.2 to study the origin of long-term variability found in its light curve.

Methods. Multi-longitude, time-resolved, photometric observations were acquired to analyze this uncommon behavior, which has been found in two newly discovered CVs.

Results. This study of SDSS J080434.20+510349.2 concerns primarily the understanding of the nature of the observed, doublehumped, light curve and its relation to a cyclic brightening that occurs during quiescence. The observations were obtained early in 2007, when the object was at about $V \sim 17.1$, about 0.4 mag brighter than the pre-outburst magnitude. The light curve shows a sinusoidal variability with an amplitude of about $0.07 \mathrm{mag}$ and a periodicity of $42.48 \mathrm{~min}$, which is half of the orbital period of the system. We observed in addition two "mini-outbursts" of the system of up to $0.6 \mathrm{mag}$, which have a duration of about 4 days each. The "mini-outburst" has a symmetric profile and is repeated in approximately every 32 days. Subsequent monitoring of the system shows a cyclical behavior of such "mini-outbursts" with a similar recurrence period. The origin of the double-humped light curve and the periodic brightening is discussed in the light of the evolutionary state of SDSS J080434.20+510349.2.
\end{abstract}

Key words. stars: dwarf novae - stars: novae, cataclysmic variables - methods: observational - techniques: photometric

\section{Introduction}

SDSS J080434.20+510349.2 (hereafter SDSS 0804) was identified as a faint $(B \sim 18 \mathrm{mag})$, short-period $\left(P_{\text {orb }}=85 \pm 3 \mathrm{~min}\right)$ cataclysmic variable by Szkody et al. (2006). These authors reported that the optical spectrum of SDSS 0804, in quiescence, shows a blue continuum with broad absorption lines from a white dwarf, which surround the double-peaked Balmer emission lines formed in an accretion disk. The spectrum is similar to the spectra of WZ Sge-type systems.

On 2006 March 4, Pavlenko et al. (2006) observed this star during a super-outburst with $V_{\max }=12.8 \mathrm{mag}$. At the end of the super-outburst, eleven echoes took place, with an interval of 2.6 days. Such post-outburst activity has been observed in only a handful of CVs, all of which are of WZ Sge type. Echoes are therefore considered to be a characteristic property of WZ Sge stars. Inspection of archive plates from Sonneberg (1923-2006) and Odessa (1968-1993) reveals only one previous outburst $(\sim 12.5 \mathrm{mag})$, which occurred in 1979

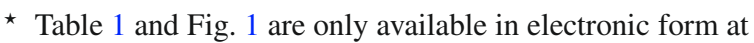
http://www. aanda.org

$\star \star$ Individual photometric measurements are only available in electronic form at the CDS via anonymous ftp to

cdsarc.u-strasbg.fr (130.79.128.5) or via

http://cdsweb.u-strasbg.fr/cgi-bin/qcat?J/A+A/486/505
}

(Pavlenko et al. 2006). Szkody et al. (2006) reported that the light curve of SDSS 0804 showed a 42.5 min periodic variability with an amplitude of $\sim 0.05 \mathrm{mag}$, which is half the spectroscopic orbital period. These double-humped light curves are observed often in the eary stages of an outburst in WZ Sge systems, and on rare occasions in quiescence. Imada et al. (2006) proposed to include the presence of double-peaked light curves in shortperiod CVs as an additional criterion for a WZ Sge-type classification. Thus, SDSS 0804 exhibits all the necessary attributes to be classified as a classical WZ Sge object: a short orbital period, infrequent and large-amplitude super-outbursts succeeded by echo outbursts, a double-humped light curve, and other features such as strong emission lines surrounded by broad absorption and long-lasting super-humps during a super-outburst.

In addition to the "standard" set of WZ Sge features, Szkody et al. (2006) detected a rapid rise in brightness of the system by $0.5 \mathrm{mag}$, at the same time as the amplitude of the $42.5 \mathrm{~min}$ variation increased to about $0.2 \mathrm{mag}$ (hereinafter named as "brightening"). A similar behavior - the large increase in brightness together with the increase in amplitude of the modulation - was first discovered by Zharikov et al. (2006) in another short period CV SDSS J123813.73-033933.0 (hereafter abbreviated as SDSS 1238), where such brightenings are cyclic. Both objects have also a similar spectral appearance in quiescence. 


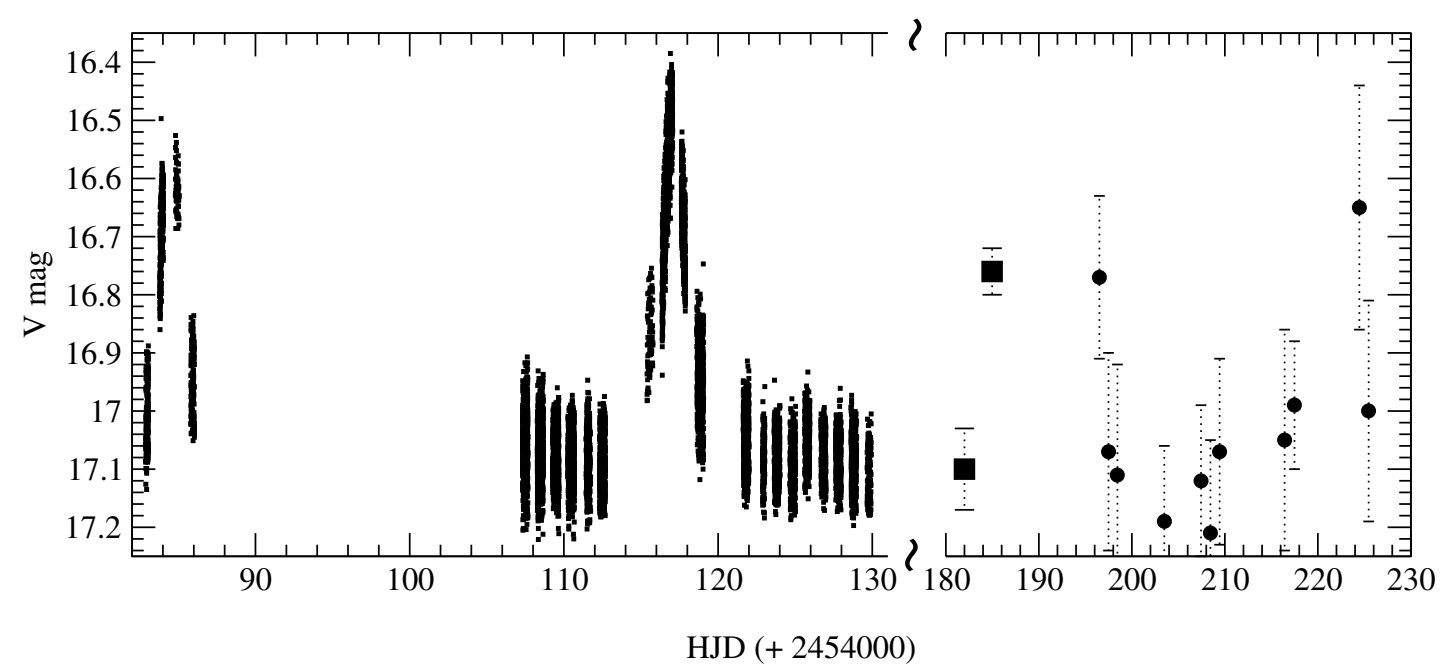

Fig. 2. The composite light curve of SDSS 0804 of data acquired throughout the campaign. The log of observations in the period HJD 2454082-2454131 is given in the Table 1. The monitoring of the system in the period HJD 2454180-2454230 is represented by the $2.1 \mathrm{~m}$ telescope BOAO (full squares) and the $0.4 \mathrm{~m}$ telescope of the Imbusch observatory (full circles) data.

Interested by the similarity between the systems, we conducted a new time-resolved photometric study of SDSS 0804 to establish the reasons behind their common nature, understand the origin of the cyclic brightening and its relation to the amplitude of the double-humped light curve. In Sect. 2, we describe our observations and data reduction. The data analysis and the results are presented in Sect. 3, while a general discussion is given in Sect. 4.

\section{Observations and data reduction}

Taking into account the long duration of the brightening and the uncertainty in a brightening cycle period of SDSS 0804, we planned and executed multi-longitude observations of this object. Time-resolved photometry is only available in electronic form at the CDS of SDSS 0804 was obtained using direct $\mathrm{CCD}$ image mode at several facilities: the $1.5 \mathrm{~m}$ and $0.84 \mathrm{~m}$ telescopes at the Observatorio Astronómico Nacional at San Pedro Mártir in Mexico; the $1.5 \mathrm{~m}$ Russian-Turkish telescope at the TUBITAK National Observatory (TUG) in Turkey; the $0.8 \mathrm{~m}$ IAC80 telescope at the Observatorio del Teide in the Canary Islands, Spain; the $2.1 \mathrm{~m}$ telescope at the Bohyunsan Optical Astronomy Observatory (BOAO) in South Korea; and the $0.4 \mathrm{~m}$ telescope at the Imbusch observatory in Galway, Ireland. The log of time-resolved observations is presented in Table 1. Several field stars as well as Landolt photometric stars were also observed.

Data reduction was performed using both ESO-MIDAS and IRAF software. The images were bias-corrected and flatfielded before aperture photometry was carried out. The errors of the differential CCD photometry were calculated from the dispersion of the magnitude of the comparison stars. The dispersion ranged from 0.01 to $0.05 \mathrm{mag}$, during the observational period HJD 2454082-2454131. The errors during HJD 2454180-2454230 were 0.05-0.1 mag for BOAO data, and $0.15-0.2 \mathrm{mag}$ for data obtained using the $0.4 \mathrm{~m}$ Imbush telescope. Calibration of the field stars, observed in the Johnson $V$ band, was obtained from the Landolt standards, and thus they became secondary standard stars. Their corresponding magnitudes are indicated in Fig. 1. A residual uncertainty in their absolute calibration may reach $\sim 0.1$ mag because of the absence of colorindex information. The magnitudes in the $R$-band were derived using the $V$ and $R$ magnitudes of the reference star marked in Fig. 1, using the USNO A2.0 catalogue (Fig. 1). The data obtained without filter (White Light: marked WL in Table 1) were transformed to the $V$-band. The light curve of the entire set of observations is presented in Fig. 2.

\section{Data analysis}

Frequent brightenings (on the timescale of a fraction of a day) were expected in the system from comparison of the behavior of SDSS 0804 (Szkody et al. 2006) with the light curve of SDSS 1238 (Zharikov et al. 2006). We show examples of brightenings of both objects side by side in the bottom panel of Fig. 3, on similar time and magnitude scales. The brightening events for SDSS 0804 and for SDSS 1238 have an almost identical behavior. The quiescent state is interrupted by a sudden and fast rise of brightness during a time corresponding to half the orbital period, with a simultaneous increase in the amplitude of the double-humped variation. For SDSS 1238, the brightness increase lasts only $\sim 3-4 \mathrm{~h}$ and repeats itself cyclically about every 8-12 h. The brightenings of SDSS 0804 last for about a similar time but there is no information on how frequently they occur prior to super-outburst.

We found repetitive brightness increases in the new observations of SDSS 0804, although their behavior was different. Firstly, we note that the object at the time of our observations had a brightness of $V \sim 17.1 \mathrm{mag}$, which is, about $0.4 \mathrm{mag}$ brighter than in the quiescent state before the 2006 superoutburst (Pavlenko et al. 2006). Earlier in 2005 (see Szkody et al. 2006), the brightness of the object was estimated to be $V \geq 17.5 \mathrm{mag}$, judging from the B-band photometry and the $(B-V) \sim 0.15$ color index calculated from the SDSS spectrum ${ }^{1}$.

Secondly, the object exhibits only two incidents of a brightness increase during the observing period corresponding to HJD 2454082-2454131, defined here as mini-outbursts to differentiate them from brightenings. The amplitudes of the minioutbursts are about $0.6 \mathrm{mag}$ and are similar to the amplitudes of the brightenings. The mini-outbursts, however, last approximately 4 days, based on comprehensive monitoring of two events. A composite profile of all mini-outbursts is presented in

1 http://www.sdss.org 

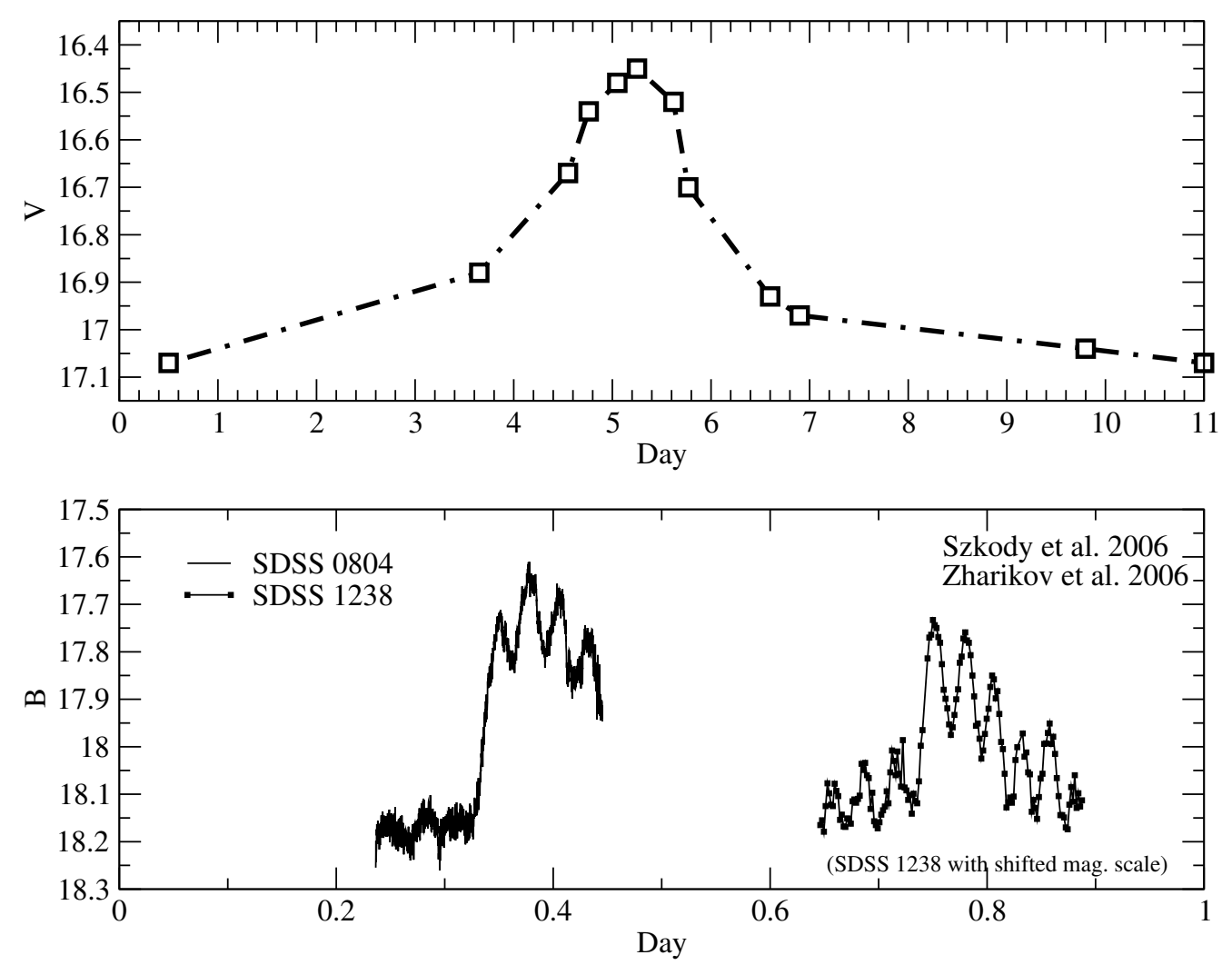

Fig. 3. The average, smoothed, composite light curve of the mini-outbursts (upper panel). Examples of brightness events in the SDSS 0804 and SDSS 1238 systems, accompanied by a change of the amplitude with half-orbital period variability (bottom panel).

the top panel of Fig. 3. Please note, that the timescales of the upper and bottom panels are different. The brightenings last only $\sim 0.2$ days. The use of the term mini-outburst is appropriate also because these events do not resemble dwarf nova outbursts: their amplitude is too small for an outburst, i.e. the total energy release is significantly smaller than usually produced in an outburst as a result of thermal instability of the accretion disk. The object probably shows two more mini-outbursts, as can be seen in the complete light curve of SDSS 0804 presented in Fig. 2. The time between the first two mini-outbursts is 32 days.

Thirdly and most importantly, we found that the object shows a double-humped light curve with constant amplitude, during all of the time that the object was observed. We do not detect any variation in the amplitude of the double humps with respect to luminosity. The brightness variation, referred to as a mini-outburst, develops slowly during 2-3 days, reaches a similar amplitude as that observed during the brightening observed by Szkody et al. (2006), but shows almost a symmetrical profile. The amplitude of the double-hump variation remains unchanged throughout the entire mini-outburst and equals the pre and postmini-outburst value.

To complete the time analysis, we separated our timeresolved observation data into two distinct categories - repose and mini-outburst, which both occurred when the system was mainly in quiescence. In a state of repose, the object flickers around $V \sim 17.1$ (see the lower panel in Fig. 4), while the minioutburst corresponds to a brightness increase in the light curve, where the brightness of the object reaches 16.5 mag at maximum (see the upper panels in Figs. 3 and 4). The data acquired during the state of repose were analyzed for periodicities using the Discrete Fourier Transform code (Deeming 1975). The power spectrum of the repose data is presented in Fig. 5 (middle panel).
The peak corresponding to the maximum power is located at $P_{\text {phot }}=42.48(2) \mathrm{min}$, which corresponds to half the orbital period of the system. The $\sim 0.07 \mathrm{mag}$ variability has a sinusoidal shape, as can be seen in the lower panel of Fig. 5, where the data are folded by the $P_{\text {phot }}=42.48(2)$ min period. The character of the light curve, before and after the mini-outburst, is completely identical. The period and phase of the periodic variations are preserved throughout the mini-outburst state. The power spectrum of the data during mini-outbursts (an example of the minioutbursts data is presented in the upper panel of the Fig. 4) shows a similar peak in frequency as that for the double-hump period, but, in this case, it is contaminated by the profile of the minioutbursts (Fig. 5, upper panel).

We assume that $P_{\text {orb }}=2 \times P_{\text {phot }}=0.05900 \mathrm{~d}$, is the true orbital period of SDSS 0804. This value is within the error range of the spectroscopic orbital period $P_{\mathrm{orb}}^{\mathrm{sp}}=0.0592(4) \mathrm{d}$, derived by Pavlenko et al. (2007). Using our estimated $P_{\text {orb }}$ value, we calculate the system mass ratio to be $q \approx 0.05$, based on its super-hump period $P_{\mathrm{sh}}=0.059713(7) \mathrm{d}$ (Pavlenko et al. 2006), and the $\varepsilon=0.18 q+0.29 q^{2}$ relation between a period excess $\varepsilon \equiv\left(P_{\text {sh }}-P_{\text {orb }}\right) / P_{\text {orb }}$ and a mass ratio $q \equiv M_{2} / M_{1}$ (Patterson et al. 2005). If this empirical relation holds for extremely short periods, then the mass of the secondary cannot exceed $0.08 M_{\odot}$.

\section{Discussion}

The cataclysmic variable SDSS 0804, since its discovery, has been proposed as a WZ Sge-type candidate, based on its short orbital period and spectral/photometric characteristics. Further evidence to support this classification was the occurrence and morphology of its 2006 super-outburst and consequent echoes (Pavlenko et al. 2006). The echoes, similar to WZ Sge 

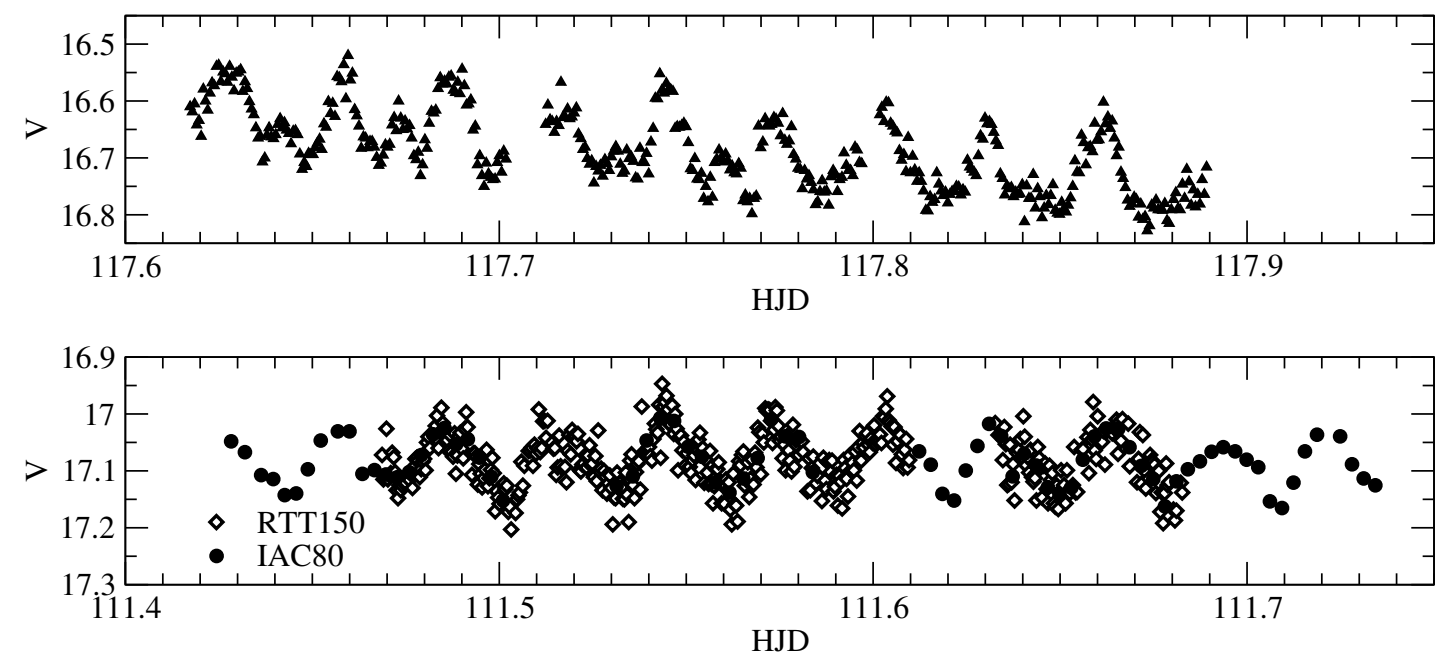

Fig. 4. Fragments of a light curve during the "mini-outburst" and "repose" states (up and low respectively).
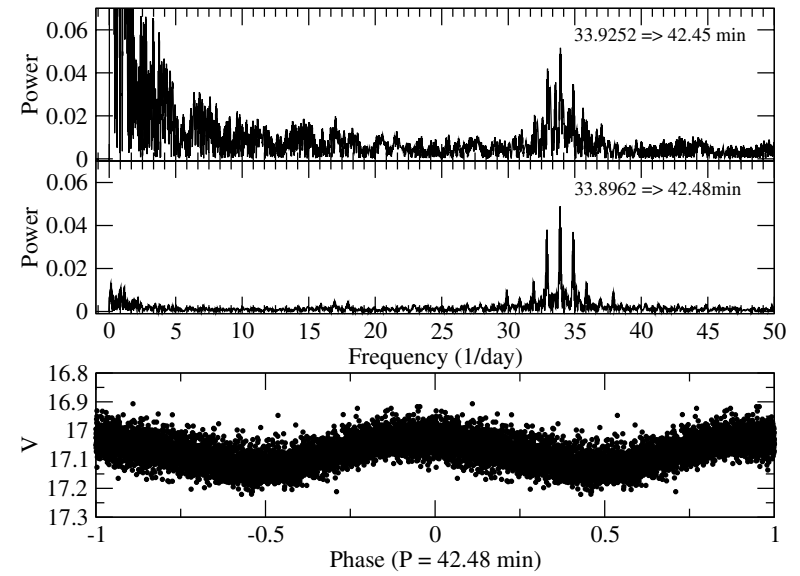

Fig. 5. The power spectrums obtained for both states: mini-outburst (top) and repose state (below). The light curve of the repose state folded by the period of $P_{\text {phot }}=42.48(2) \mathrm{min}=1 / 2 P_{\text {orb }}$ ( bottom panel).

(Patterson et al. 2002) and EG Cnc (Kato et al. 2004, and references therein), are considered as an exclusive property of the WZ Sge class among dwarf novae. Super-humps with the period of $0.059713 \mathrm{~d}$ were also detected during the super-outburst, which led to a low mass ratio estimate of $q \sim 0.05$. During our observations taken about one year after its super-outburst, SDSS 0804 remained brighter, by about $0.4 \mathrm{mag}$, than it was before the March 2006 event. A similar increase in the quiescence level after the super-outburst, was observed in another WZ Sgetype system Al Com (Nogami et al. 1997).

In addition to classical WZ Sge properties, SDSS 0804 exhibited a variability that was almost identical to that observed for SDSS 1238. First, there was the persistent double-humped light curve in quiescence, which had however variable amplitude. Then, there were the cyclic luminosity increases of only a half magnitude.

Various models (see Patterson et al. 2002; Imada et al. 2006, and ref. therein) have been proposed to explain the doublehumped light curves in WZ Sge systems, among which the 2:1 resonance (Lin \& Papaloizou 1979; Osaki \& Meyer 2002; Kunze \& Speith 2005) in systems with mass ratio $q \leq 0.1$ is favoured. If this 2:1 resonance is responsible for the doublehumped light curves, then we have to account for the difference between "classical" WZ Sge-type systems (WZ Sge,

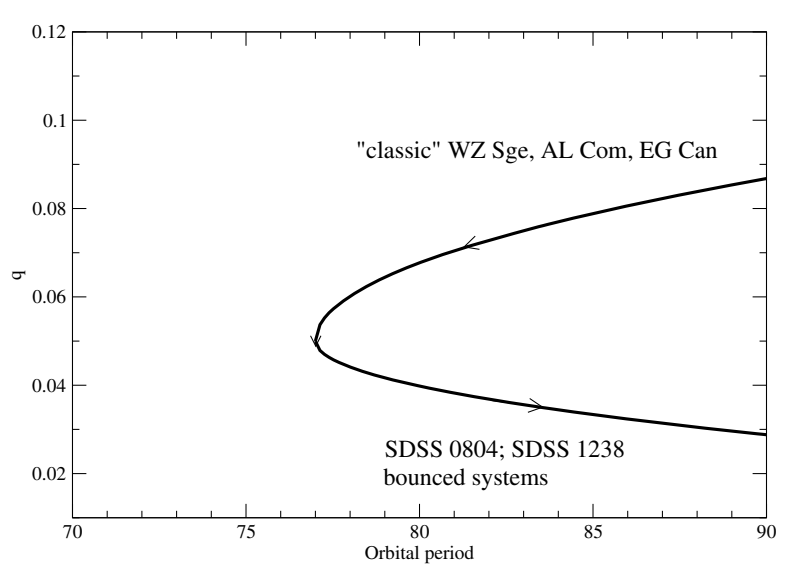

Fig. 6. The schematic evolution state of "classical" WZ-Sge system and "non-classical" SDSS 0804 and SDSS 1238.

ALCom, EGCnc) and the newly SDSS-discovered objects (i.e. SDSS 0804 and SDSS 1238), and explain why they undergo cyclical brightenings during quiescence. According to the 2:1 resonance model the rim of the disk expands and reaches the 2:1 resonance region during the super-outburst in "classical" WZ Sge systems. If SDSS 0804 and SDSS 1238 contain less massive secondaries than "classical" WZ Sge systems, it is possible that the radius of the accretion disk in these systems is continually reaching of 2:1 resonance radius. Less massive secondaries put SDSS 0804 and SDSS 1238 as "period bounce" systems, i.e. close binaries which have reached the period limit $\sim 77$ min boundary and have turned around (Barker \& Kolb 2003), as opposed to "classical" WZ Sge systems, which are still evolving towards an orbital period minimum (see Fig. 6). Steeghs et al. 2007 determined the mass ratio $M_{2} / M_{1}$ for the components of WZ Sge itself to be $0.075<q<0.101$. Their inferred donor mass $M_{2}=0.078 \pm 0.06 M_{\odot}$ corresponds to an L2-type star and according to Knigge (2006), the system still evolves toward its period minimum. Using a large range of masses for the white dwarf in SDSS $08040.6 M_{\odot}<M_{1}<$ $1.4 M_{\odot}$ and our estimate of $q=0.05$ we calculate that the secondary mass is in the range $0.03 M_{\odot}<M_{2}<0.07 M_{\odot}$, which makes it more likely to be a post-period minimum system.

The observations of SDSS 1238 show cyclical or quasiperiodic brightenings with a sudden increase in amplitude of the 
double-hump curve (Zharikov et al. 2006). The same is probably true for the pre-outburt behavior of SDSS 0804. The cyclical nature of the brightenings suggests that the mass-transfer rate varies cyclically too. If this is the case, even a small increase in the mass transfer rate of a system will cause an expansion of the accretion disk, with a rapid increase in the brightness of the system and a long extended tail in the decay phase (Ichikawa \& Osaki 1992). In combination with the disk-size increase, the two-armed spiral dissipation pattern will form and emerge as a double-humped light curve (Kunze \& Speith 2005).

After the 2006 super-outburst, the behavior of SDSS 0804 in quiescence has qualitatively changed. Firstly, nine months after super-outburst the system has still not descended to its preoutburst quiescent level, but remains about 60 percent brighter than it was before. Secondly, during the entire duration of our observational campaign, the system displayed a double-humped light curve of approximately similar amplitude. Thirdly, the cyclical brightenings have changed significantly. The timescale of this change exceeds significantly that of the brightenings observed for SDSS 1238 (Zharikov et al. 2006), and that observed for SDSS 0804 by Szkody et al. (2006). The recurrence time, compared to that of SDSS 1238, is incompatibly longer. The shape is different and the brightening, or mini-outburst to differentiate it from conventional brightenings, is similar to that of a normal outburst in SU UMa systems, but it has significantly lower amplitude, when compared to the typical amplitude in DN systems ranging from 2 to $6 \mathrm{mag}$, or is as a "stunted" outburst such as that observed in some nova-like cataclysmic variables (Honeycutt 2001). This is a new phenomenon that has not been observed before in other WZ Sge-like systems in quiescence. Finally and most importantly, the amplitude of the double-humped variation does not depend on the mini-outburst occurrence.

Another mechanism is required to explain the mini-outbursts in addition to the double-humped light curve and the brightenings prior to the super-outburst. We find that such a mechanism could be an irradiation of the accretion disk as a result of the super-outburst. The small amplitude post-eruption outbursts with an amplitude of only $\sim 0.5 \mathrm{mag}$ were predicted by Hameury et al. (1999). These authors modeled the timedependent behavior of irradiated accretion disks in dwarf novae and post novae following an outburst. The contribution of irradiation by the white dwarf to the inner parts of the accretion disk was found to cause small outbursts, followed immediately by normal outbursts, or even a super-outburst. Since such outbursts had been not observed before, Hameury et al. (1999) concluded, that either the inner disk was evaporated or the efficiency of the irradiating flux from the white dwarf was lower than expected. According to their models, these small outbursts, or mini-outbursts, as we call them to distinguish them from normal outbursts, start, however, as inside-out outbursts, which die out before they reach half the radius of the accretion disk, unable to propagate across the entire disk. This leaves the outer parts of the disk intact, where the 2:1 resonance occurs. In the case of SDSS 0804, the internal parts of the disk have probably not been destroyed during the super-outburst and hence, it is possible to observe the prolonged effect of irradiation in a dwarf nova directly. Furthermore, the irradiation of the disk might be the same mechanism that produces the echos appearing after the super-outburst in SDSS 0804 and some other WZ Sge systems. Because of the peculiar mass ratio of a period-bounce system, the mass-transfer rates and, the accretion disk size, we observed the effect of irradiation for a long period of time.

\section{Conclusions}

We observed SDSS 0804 almost a year after it underwent a super-outburst. The system exhibits all the attributes of a WZ Sge-type system and, in addition, shows low-amplitude cyclical mini-outburst activity, which causes them to become brighter than during the pre-outburst quiescent level. We identify these mini-outbursts as the small inside-out outbursts predicted by Hameury et al. (1999) as a result of an irradiation of a disk by a powerful super-outburst. The mini-outbursts differ from the brightenings observed previously in SDSS 0804 and SDSS 1238. The brightenings have similar amplitude as the mini-outbursts but show a different temporal behavior and therefore a smaller energy output. We suggest that variable mass transfer produces the brightenings, and directly influences the $2: 1$ resonance effect, which determines the amplitude of the double-hump light curve. On the other hand, the mini-outbursts are of a sporadic nature as a result of irradiation of the accretion disk and are not related to the amplitude of the double humps. We argue that both of these CVs have probably evolved beyond the period limit, and hence, are members of long sought, elusive bounced-back systems, and therefore differ from other WZ Sge systems.

The new time-resolved spectral observations of these system with high signal/noise ratio obtained during quiescence, would be useful help us understand the accretion-disk structure changes that correspond to the re-brightening phenomena. In addition, the numeric simulation of the accretion disk dynamic in $2: 1$ resonance can help us to understand the dynamics of the evolution of spiral-armed structures in accretion disks and their observational properties.

Acknowledgements. This work was partially supported by PAPIIT IN101506 and CONACYT 48493 projects. V.N. acknowledges support of IRCSET under their basic research programme and the support of the HEA funded CosmoGrid project. We wish to thank Prof. Mike Redfern for help with the observations at the Imbusch observatory. We thank the anonymous referee, for comments that led to an improved presentation of the paper.

\section{References}

Barker, J., \& Kolb, U. 2003, MNRAS, 340, 623

Deeming, T. J. 1975, Ap\&SS, 36, 137

Hameury, J.-M., Lasota, J.-P., \& Dubus, G. 1999, MNRAS, 303, 39

Honeycutt, R. K. 2001, PASP, 113, 473

Ichikawa, S., \& Osaki, Y. 1992, PASJ, 44, 15

Imada, A., Kubota, K., Kato, T., et al. 2006, PASJ, 58, L19

Lin, D. N. C., \& Papaloizou, J. 1979, MNRAS, 186, 799

Kato, T., Nogami, D., Matsumoto, K., \& Baba, H. 2004, PASJ, 56, 109

Knigge, C. 2006, MNRAS, 373, 484

Kunze, S., \& Speith, R. 2005, The Astrophysics of Cataclysmic Variables and Related Objects, ed. J.-M. Hameury, \& J.-P. Lasota (San Francisco: ASP), Proc. ASP Conf., 330, 389

Nogami, D., Kato, T., Baba, H., et al. 1997, ApJ, 490, 840

Osaki, Y., \& Meyer, F. 2002, A\&A, 383, 574

Patterson, J., Masi, G., Richmond, M. W., et al. 2002, PASP, 114, 721

Patterson, J., Kemp, J., Harvey, D. A., et al. 2005, PASP, 117, 1204

Pavlenko, E. P., Shugarov, S. Yu., Katysheva, N. A., et al. 2006, Binary Stars as Critical Tools and Tests in Contemporary Astrophysics, International Astronomical Union. Symp., 240, held 22-25 August, 2006 in Prague, Czech Republic, 89

Pavlenko, E. P., Shugarov, S. Yu., Katysheva, N. A., et al. 2007, 15th European Workshop on White Dwarfs ASP Conf. Ser., 372, proceedings of the conference held 7-11 August, 2006 in Leicester, UK, ed. R. Napiwotzki, \& M. R. Burleigh (San Francisco: ASP), 511

Schreiber, M. R., \& Gänsicke, B. T. 2001, A\&A, 375, 937

Steeghs, D., Howell, S. B., Knigge, C., et al. 2007, ApJ, 667, 442

Szkody, P., Henden, A., Agüeros, M., et al. 2006, AJ, 131, 973

Warner, B. 1995, Cataclismic Variable Stars (Cambridge University Press)

Zharikov, S. V., Tovmassian, G. H., Napiwotzki, R., et al. 2006, A\&A, 449, 645 
S. V. Zharikov et al.: Cyclic brightening in the short-period CV SDSS 0804, Online Material $p 1$

Table 1. Log of time-resolved observations of SDSS J080434.20+510349.2.

\begin{tabular}{llllcc}
\hline \hline $\begin{array}{l}\text { Date } \\
\text { Photometry }\end{array}$ & $\begin{array}{l}\text { HJD Start+ } \\
2454000\end{array}$ & Telescope & Band & $\begin{array}{c}\text { Exp. time } \\
\text { Num. of integrations }\end{array}$ & Duration \\
\hline 12 Dec. 2006 & 82.888 & $1.5 \mathrm{~m} / \mathrm{SPM}$ & $R$ & $180 \mathrm{~s} \times 101$ & $4.20 \mathrm{~h}$ \\
13 Dec. 2006 & 83.794 & $1.5 \mathrm{~m} / \mathrm{SPM}$ & $R$ & $180 \mathrm{~s} \times 101$ & $5.77 \mathrm{~h}$ \\
14 Dec. 2006 & 84.804 & $1.5 \mathrm{~m} / \mathrm{SPM}$ & $R$ & $180 \mathrm{~s} \times 119$ & $5.76 \mathrm{~h}$ \\
15 Dec. 2006 & 85.802 & $1.5 \mathrm{~m} / \mathrm{SPM}$ & $R$ & $120 \mathrm{~s} \times 129$ & $5.81 \mathrm{~h}$ \\
\hline 6 Jan. 2007 & 107.317 & $1.5 \mathrm{~m} / \mathrm{RTT150}$ & $V$ & $120 \mathrm{~s} \times 133$ & $8.32 \mathrm{~h}$ \\
7 Jan. 2007 & 108.276 & $1.5 \mathrm{~m} /$ RTT150 & $V$ & $120 \mathrm{~s} \times 128$ & $9.31 \mathrm{~h}$ \\
8 Jan. 2007 & 109.278 & $1.5 \mathrm{~m} / \mathrm{RTT} 150$ & $V$ & $120 \mathrm{~s} \times 143$ & $9.48 \mathrm{~h}$ \\
9 Jan. 2007 & 110.263 & $1.5 \mathrm{~m} / \mathrm{RTT} 150$ & $V$ & $120 \mathrm{~s} \times 135$ & $10.03 \mathrm{~h}$ \\
10 Jan. 2007 & 111.468 & $1.5 \mathrm{~m} / \mathrm{RTT} 150$ & $V$ & $120 \mathrm{~s} \times 135$ & $5.11 \mathrm{~h}$ \\
11 Jan. 2007 & 112.325 & $1.5 \mathrm{~m} / \mathrm{RTT} 150$ & $V$ & $120 \mathrm{~s} \times 135$ & $8.57 \mathrm{~h}$ \\
\hline 10 Jan. 2007 & 111.428 & $0.8 \mathrm{~m} / \mathrm{IAC} 80$ & $\mathrm{WL}$ & $120 \mathrm{~s} \times 135$ & $7.44 \mathrm{~h}$ \\
14 Jan. 2007 & 115.390 & $0.8 \mathrm{~m} / \mathrm{IAC} 80$ & $\mathrm{WL}$ & $120 \mathrm{~s} \times 135$ & $9.19 \mathrm{~h}$ \\
15 Jan. 2007 & 116.369 & $0.8 \mathrm{~m} / \mathrm{IAC} 80$ & $\mathrm{WL}$ & $120 \mathrm{~s} \times 135$ & $9.67 \mathrm{~h}$ \\
\hline 15 Jan. 2007 & 116.745 & $0.84 \mathrm{~m} / \mathrm{SPM}$ & $V$ & $180 \mathrm{~s} \times 101$ & $7.34 \mathrm{~h}$ \\
16 Jan. 2007 & 117.617 & $0.84 \mathrm{~m} / \mathrm{SPM}$ & $V$ & $180 \mathrm{~s} \times 119$ & $6.52 \mathrm{~h}$ \\
17 Jan. 2007 & 118.624 & $0.84 \mathrm{~m} / \mathrm{SPM}$ & $V$ & $120 \mathrm{~s} \times 129$ & $10.53 \mathrm{~h}$ \\
20 Jan. 2007 & 121.629 & $0.84 \mathrm{~m} / \mathrm{SPM}$ & $V$ & $180 \mathrm{~s} \times 101$ & $8.89 \mathrm{~h}$ \\
21 Jan. 2007 & 122.881 & $0.84 \mathrm{~m} / \mathrm{SPM}$ & $V$ & $180 \mathrm{~s} \times 101$ & $3.55 \mathrm{~h}$ \\
22 Jan. 2007 & 123.623 & $0.84 \mathrm{~m} / \mathrm{SPM}$ & $V$ & $180 \mathrm{~s} \times 101$ & $9.20 \mathrm{~h}$ \\
23 Jan. 2007 & 124.659 & $0.84 \mathrm{~m} / \mathrm{SPM}$ & $V$ & $180 \mathrm{~s} \times 119$ & $8.81 \mathrm{~h}$ \\
24 Jan. 2007 & 125.617 & $0.84 \mathrm{~m} / \mathrm{SPM}$ & $V$ & $120 \mathrm{~s} \times 129$ & $7.90 \mathrm{~h}$ \\
\hline 25 Jan. 2007 & 126.671 & $1.5 \mathrm{~m} / \mathrm{SPM}$ & $V$ & $180 \mathrm{~s} \times 101$ & $7.56 \mathrm{~h}$ \\
26 Jan. 2007 & 127.626 & $1.5 \mathrm{~m} / \mathrm{SPM}$ & $V$ & $180 \mathrm{~s} \times 101$ & $8.62 \mathrm{~h}$ \\
27 Jan. 2007 & 128.623 & $1.5 \mathrm{~m} / \mathrm{SPM}$ & $V$ & $180 \mathrm{~s} \times 119$ & $8.66 \mathrm{~h}$ \\
28 Jan. 2007 & 129.672 & $1.5 \mathrm{~m} / \mathrm{SPM}$ & $V$ & $120 \mathrm{~s} \times 129$ & $5.90 \mathrm{~h}$ \\
\hline
\end{tabular}


S. V. Zharikov et al.: Cyclic brightening in the short-period CV SDSS 0804, Online Material p 2

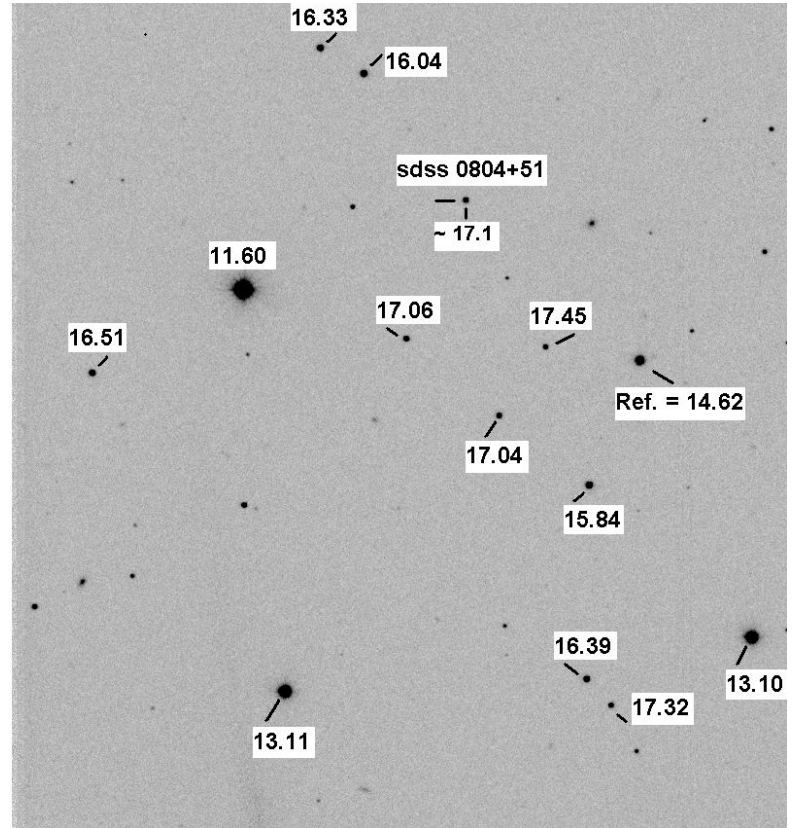

Fig. 1. The field of SDSS 0804 observed using the RTT150 telescope. The north is at the top of the image and the east is at the left. The image size is $\sim 6.5 \times 6.5$ arcmin. The object and the secondary standard stars are indicated. The $V$-band magnitudes of the secondary standard stars are marked. 